\title{
Profundidade da amostragem de solo e de raízes e índice de infestação de Oryzophagus oryzae (Costa Lima, 1936) (Coleoptera: Curculionidae) em cultivares de arroz
}

\author{
Sampling depth of soil and roots and Oryzophagus oryzae (Costa Lima, 1936) \\ (Coleoptera: Curculionidae) infestation index in rice cultivars
}

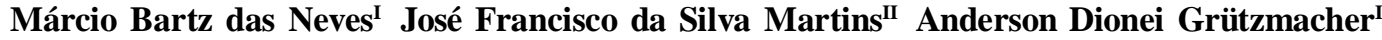 \\ Crislaine Alves Barcellos de Lima $^{\mathrm{I}}$ Germano Tessmer Büttow ${ }^{\mathrm{I}}$
}

\section{RESUMO}

Oryzophagus oryzae é o inseto-praga chave da cultura do arroz irrigado por inundação na Região Sul do Brasil. Suas larvas, conhecidas por bicheira-da-raiz, causam perdas significativas de produtividade quando danificam o sistema radicular das plantas de arroz. No monitoramento da população larval de $\boldsymbol{O}$. oryzae em arrozais, ou em levantamentos em experimentos, atualmente são empregadas amostras padrão de solo e raízes, com $8,5 \mathrm{~cm}$ de profundidade e $10 \mathrm{~cm}$ de largura, desintegradas por submersão e agitamento em peneira com água, para a contagem de larvas. A visualização de larvas, principalmente de $1^{\circ}$ e $2^{\circ}$ instar, tornase difícil na suspensão formada pelo solo das amostras com tais dimensões e a água do interior das peneiras. Assim sendo, foi realizado um experimento para determinar uma profundidade de amostragem que viabilize a captura e a visualização de um maior número possível de larvas, num menor volume possivel de solo, em diferentes cultivares de arroz. O experimento foi instalado num Planossolo Háplico, no delineamento de quadrado latino, e incluiu como tratamentos seis cultivares de arroz ('BRS Atalanta'; 'BRS Firmeza'; 'BRS Ligeirinho'; 'BRS Querência'; 'BRS Sinuelo $C L$ '; 'IRGA 417') e três profundidades (3, 6 e 9cm) de retirada de amostras de solo e raizes, todas com $10 \mathrm{~cm}$ de diâmetro. De imediato à retirada de amostras, foi registrado o número de larvas. Posteriormente, em avaliações periódicas, foi registrado o número de adultos emergidos de amostras mantidas intactas em baldes plásticos com lâmina de água de 13, 16 e $19 \mathrm{~cm}$, cobertos por tecido telado. Ocorreu interação significativa entre profundidades de amostragem e as cultivares 'BRS Sinuelo $C L$ ' e 'BRS Querência' quanto ao índice de infestação larval e de adultos de O. oryzae, respectivamente. No caso de ambas as variáveis, o indice na profundidade de $6 \mathrm{~cm}$ foi significativamente maior do que na de $3 \mathrm{~cm}$, mantendo-se intermediário na de $9 \mathrm{~cm}$. Concluiu-se que, num Planossolo Háplico, amostras de solo e de raízes de arroz retiradas à profundidade de $6 \mathrm{~cm}$ são mais apropriadas à captura e à visualização de larvas de O. oryzae, na suspensão (solo e água) formada nas peneiras usadas para a sua contagem, e à obtenção de adultos desse inseto.

Palavras-chave: Oryza sativa, gorgulho-aquático, contagem de insetos, método, manejo integrado de pragas.

\section{ABSTRACT}

Oryzophagus oryzae is the key insect pest in flooded rice fields in southern Brazil. Their larvae, known as rice water weevil cause significant yield losses when they cut the roots of rice plants. For the monitoring and survey of the larval population of $\boldsymbol{O}$. oryzae, on rice fields and rice trials, respectively, are currently used standard samples of soil and roots, with $8.5 \mathrm{~cm}$ deep and $10 \mathrm{~cm}$ wide, blown away by submersion and shaking in a sieve with water for the counting of larvae. The visualization of larvae, especially the 1st and 2nd instars, becomes difficult in the suspension formed by the soil samples with these dimensions and the water inside the sieves. Thus, an experiment was conducted in a Typic Albaqualf soil according to the Latin square design to determine a sampling depth of soil and roots that makes possible the capture and display a larger number of larvae in a smallest possible volume of soil, in different rice cultivars. The treatments consisted of six rice cultivars ('BRS Atalanta'; 'BRS Firmeza'; 'BRS Ligeirinho'; 'BRS Querência'; 'BRS Sinuelo CL'; 'IRGA 417') and three depths $(3,6$ and $9 \mathrm{~cm})$ for removing samples of soil and roots, every $10 \mathrm{~cm}$ diameter. In the immediate withdrawal of samples,

'Departamento de Fitossanidade, Faculdade de Agronomia Eliseu Maciel, Universidade Federal de Pelotas (UFPel), Pelotas, RS, Brasil.

"Embrapa Clima Temperado, BR 392, km 78, CP 403, 96010-971, Pelotas, RS, Brasil. E-mail: jose.martins@cpact.embrapa.br. Autor para correspondência. 
it was announced the number of larvae. Subsequently, in periodic evaluations, it was announced the number of adults emerged from samples kept intact in plastic buckets with water depth of 13, 16 and $19 \mathrm{~cm}$, covered by screened mesh fabric. It was detected a significant interaction between sampling depths and the rice cultivars 'BRS Sinuelo CL' and 'BRS Querência' in the rate of infestation of larvae and adults of $\boldsymbol{O}$. oryzae, respectively. In the case of both variables the number of insects per sample was significantly higher at $6 \mathrm{~cm}$ depth than at $3 \mathrm{~cm}$, an intermediate in $9 \mathrm{~cm}$. It was concluded that in Typic Albaqualf soil, samples (soil and rice roots) taken from a depth of $6 \mathrm{~cm}$ are more effective to capture and visualize larvae of $\boldsymbol{O}$. oryzae in suspension (soil and water) formed in the sieves used for counting larvae, and also for the collection of adults of this insect.

Key words: Oryza sativa, rice water weevil, insect count, method, integrated pest management.

\section{INTRODUÇÃO}

Oryzophagus oryzae é o inseto-praga mais prejudicial à cultura do arroz irrigado por inundação no Sul do Brasil, podendo causar perdas de até $18 \%$ na produtividade (MARTINS \& PRANDO, 2004). Em algumas circunstâncias, o inseto adulto, conhecido por gorgulho-aquático, pode destruir quantidade expressiva de plântulas, principalmente em cultivos de arroz pré-germinado (MARTINS et al., 2004). Entretanto, são as larvas, conhecidas por bicheira-daraiz, que causam os danos mais severos à cultura. Ao cortarem as raízes de arroz, provocam redução da absorção de nutrientes e, consequentemente, prejudicam o desenvolvimento das plantas (CARBONARI et al., 2000; MOREIRA, 2002).

Algumas práticas culturais adotadas nos arrozais contribuem para reduzir a população e os danos de o. oryzae (MARTINS \& CUNHA, 2007). Em determinadas circunstâncias, porém, não evitam a ocorrência de níveis de infestação larval economicamente prejudiciais à cultura, tornando necessário o uso de inseticidas (MARTINS \& CUNHA, 2007). O controle químico se mantém como o método mais usado, podendo, no caso das larvas, ser feito por meio de inseticidas granulados aplicados diretamente na água de irrigação (MARTINS et al., 2004; GRÜTZMACHER et al., 2008). As aplicações, porém, geralmente são realizadas sem considerar princípios do manejo integrado de pragas - MIP, como o monitoramento prévio da população larval nos arrozais (MARTINS \& CUNHA, 2007).

A intensificação do monitoramento da população larval de $\boldsymbol{O}$. oryzae, que deve ser praticado a partir do 10 dia pós-inundação da lavoura e, se necessário, repetido a intervalos de cinco a sete dias até o início de diferenciação da panícula - IDP, é essencial por indicar a real necessidade de aplicar inseticidas (MARTINS \& CUNHA, 2007). Justificamse tais aplicações somente se houver risco de ser atingido um nível de infestação superior a 5 larvas/ amostra (CONGRESSO, 2007).

$\mathrm{Na}$ literatura, há escassez de informação sobre métodos para monitoramentos da bicheira-daraiz, caracterizada como praga de solo. A mobilidade horizontal e vertical dessas pragas no solo é um dos fatores que dificultam o seu manejo, principalmente, a amostragem (SILVA, 1998). Estudo sobre distribuição de Sternechus subsignatus Boheman, 1836 (Coleoptera: Curculionidae), indicou que as larvas, ainda no 50 ínstar, aprofundam-se no solo para hibernar em câmaras construídas à profundidade $20 \mathrm{~cm}$ (HOFFMANNCAMPO et al., 1989). Outro estudo sobre a distribuição vertical no solo das larvas desse inseto, em áreas de soja e milho, indicou sua presença até a profundidade de $20 \mathrm{~cm}$, sendo um maior número encontrado em profundidades de 5 e $10 \mathrm{~cm}$ (SILVA, 1998). A compactação do solo, entre outros fatores associados a chuvas, seca, temperatura e à ação de inimigos naturais foi destacada por dificultar a mobilidade das larvas de $\boldsymbol{S}$. subsignatus. Análise da distribuição de Phyllophaga cuyabana Moser, 1919 (Coleoptera: Melolonthidae), em solo de cultivo de soja, indicou que larvas de $1^{\circ}$ instar concentraram-se entre 5 e $10 \mathrm{~cm}$ de profundidade, podendo aprofundar-se até $30 \mathrm{~cm}$ na fase final de crescimento (OLIVEIRA et al., 2009).

As larvas de $\boldsymbol{O}$. oryzae, por serem ápodas e permanecerem afixadas às raízes de arroz, por meio de espiráculos (MARTINS et al. 2004), não apresentam a mobilidade de outros insetos-praga no solo. Assim sendo, a amostragem poderia ser realizada a uma profundidade padrão, até onde as larvas se fixam às raízes, independentemente da interferência de condições climáticas e do solo, e do estágio fenológico das plantas de arroz, diferentemente do praticado para outros insetos-praga, que possuem mobilidade no perfil do solo.

O método mais utilizado para monitorar a população larval de $\boldsymbol{O}$. oryzae em lavouras comerciais, assim como para levantamentos em áreas experimentais, consiste de uma adaptação da técnica de TUGWELL \& STEPHEN (1981), aplicada à espécie Lisshoroptrus oryzophilus Kuschel (Coleoptera: Curculionidae). Tal método inclui a coleta de amostras-padrão de solo e raízes, usando uma seção de tubo rígido de PVC com $10 \mathrm{~cm}$ de diâmetro e $20 \mathrm{~cm}$ de altura, aprofundando-a $\pm 8,5 \mathrm{~cm}$ no solo; de imediato, as amostras são agitadas sob água em uma peneira com fundo de tela de náilon (malha de $1 \mathrm{~mm}^{2}$ ) para liberação e contagem das larvas (MARTINS \& CUNHA, 2007). Um aspecto crítico que 
pode interferir na avaliação do índice de infestação de O. oryzae por meio dessa técnica de amostragem é a visualização das larvas, principalmente de $1^{\circ}$ e $2^{\circ}$ instar, na suspensão (mistura de solo com água) que se forma no interior das peneiras. Quanto maior a profundidade da amostragem, mais material sólido poderá ser aportado à mistura, dificultando a visualização das larvas. Dependendo da classe de solo, também poderá haver dificuldade para visualizar as larvas, principalmente, se a amostragem atingir camadas mais adensadas. Nesse sentido, há indicação de que a profundidade de amostragem em solos argilosos e arenosos, visando à contagem de larvas de L. oryzophilus, deve ser de 5 a $7,5 \mathrm{~cm}$ e de 7,5 a $10 \mathrm{~cm}$, respectivamente (JOHNSON et al., 2006).

A definição sobre a profundidade de amostragem de solo e raízes destinadas à contagem de larvas de $\boldsymbol{O}$. oryzae ainda depende da finalidade da avaliação. Em processos de monitoramento em lavouras comerciais, basicamente é utilizado o número de larvas/ amostra (CONGRESSO, 2007). Por outro lado, em trabalhos de pesquisa, como nos inerentes à resistência de cultivares ao inseto, além do número de larvas, são requeridos, também, dados complementares sobre a população de adultos que emergem de amostras mantidas intactas em condição de submersão (SILVA et al., 2003). Assim sendo, foi realizado um experimento com o objetivo de determinar uma profundidade de amostragem que viabilize a obtenção e a visualização de um maior número possível de larvas e/ou adultos de O. oryzae, incluindo a avaliação do grau de influência de diferentes cultivares no processo.

\section{MATERIAL E MÉTODOS}

Um experimento foi instalado na Estação Experimental Terras Baixas da Embrapa Clima Temperado, em Capão do Leão, RS, num Planossolo Háplico, sendo adotado o sistema de transplante de mudas (CONGRESSO, 2007) e o delineamento de quadrado latino, incluindo como tratamentos seis cultivares de arroz irrigado ('BRS Atalanta'; 'BRS Firmeza'; 'BRS Ligeirinho'; 'BRS Querência'; 'BRS Sinuelo CL'; 'IRGA 417') e três profundidades (3, 6 e $9 \mathrm{~cm})$ de retirada de amostras de solo e raízes, todas com $10 \mathrm{~cm}$ de diâmetro.

Inicialmente, em 21 de outubro de 2009, foi implementada a produção de mudas, em casa telada, por meio da distribuição de sementes de cada cultivar em quatro bandejas plásticas $(27 \times 53 \mathrm{~cm})$ contendo 128 células preenchidas à altura de $2,5 \mathrm{~cm}$ com um Planossolo Háplico peneirado. Cada célula, de imediato à colocação de cinco sementes, foi coberta com uma camada adicional de $1 \mathrm{~cm}$ do mesmo solo, sendo as bandejas irrigadas diariamente. Aos dez dias após a emergência das plântulas (15 de novembro de 2009), quando atingiram $10 \mathrm{~cm}$ de altura, foi realizado um desbaste, sendo mantida apenas uma planta/célula. $\mathrm{O}$ preparo do solo (aração, gradagem e aplainamento) foi realizado mecanicamente, sendo construída uma taipa ao entorno do experimento. $\mathrm{O}$ transplante foi realizado manualmente em 05 de dezembro de 2009 num solo coberto por uma lâmina de água de $2 \mathrm{~cm}$ de espessura, sendo colocadas 30 plântulas individualizadas, com altura entre 15 a $20 \mathrm{~cm}$ por parcela experimental de $1,2 \mathrm{~m}^{2}$ (cinco fileiras de seis plantas equidistantes $20 \mathrm{~cm}$ ). Entre parcelas, na cabeceira e na lateral, foi mantido o espaçamento de $1 \mathrm{~m}$. A partir da data do transplante até 12 dias após (17 de dezembro de 2009), a lâmina de água foi aumentada progressivamente, sendo estabilizada em $15 \mathrm{~cm}$ de espessura. Em 19 de janeiro de 2009, aos 33 dias pós-estabilização da lâmina de água, por meio do método indicado por MARTINS \& CUNHA (2007), foram coletadas aleatoriamente 12 amostras padrão de solo e raízes na $1^{\text {a }}$ e na $5^{\underline{a}}$ fileira de planta de cada parcela. Todas as amostras foram retiradas à profundidade de $9 \mathrm{~cm}$ e depois cortadas formando subconjuntos de quatro amostras, cada um correspondendo a uma profundidade de amostragem $(3,6$ ou $9 \mathrm{~cm})$. Logo após a coleta, essas amostras foram desintegradas em água para a contagem de larvas.

Em 25 de janeiro de 2009, aos 39 dias pósestabilização da lâmina de água, de acordo com as três profundidades de amostragem, foram retiradas aleatoriamente mais 12 amostras padrão de solo e raízes, porém, na $2^{\mathrm{a}}$ e na $4^{\mathrm{a}}$ fileira de planta de cada parcela. Essas quatro subamostras inerentes a cada profundidade de amostragem foram mantidas intactas para registro da emergência de adultos de $\boldsymbol{O}$. oryzae. Para tal, as amostras foram agrupadas em baldes plásticos $(30 \mathrm{~cm}$ de diâmetro x $50 \mathrm{~cm}$ de altura), submersas numa lâmina de água com espessura de $10 \mathrm{~cm}$, acima do topo da amostra (portanto de 13,16 e $19 \mathrm{~cm}$, respectivamente) e em seguida cobertas por tecido telado. $\mathrm{O}$ número de adultos foi registrado em intervalos de três dias, até 30 dias pós-início dos levantamentos, época em que cessou a emergência do inseto. A água nos baldes foi trocada por ocasião de cada levantamento para manter uma adequada oxigenação e translucidez necessárias à sobrevivência e à visualização do inseto. As avaliações foram feitas preferencialmente em horários (final da manhã ou início da tarde) com temperatura e luminosidade favoráveis ao deslocamento do inseto da água dos baldes ao topo das plantas e ao tecido telado, facilitando a contagem. 
Os dados obtidos (x) foram transformados em $\sqrt{x}$ para a análise de variância (ANOVA), sendo as médias comparadas pelo teste de Tukey em nível de 5\% de probabilidade. Nas análises, foi utilizado o programa estatístico "ASSISTAT" versão 7.5 (SILVA, 2008).

\section{RESULTADOS E DISCUSSÃO}

No experimento, foram detectadas, em média, 6,1 larvas de $\boldsymbol{O}$. oryzae/amostra de solo e raízes, superior a 5 larvas/amostra, que é o índice de infestação mínimo indicativo da necessidade de adoção de medidas de controle do inseto (CONGRESSO, 2007).

As cultivares de arroz não diferiram significativamente quanto ao número de larvas de O. oryzae obtido em cada profundidade de amostragem $(3,6$ ou $9 \mathrm{~cm})$. Por outro lado, ocorreu interação entre profundidade de amostragem e cultivar quanto ao número de larvas, porém, apenas na 'BRS Sinuelo CL'. Nessa cultivar, o número de larvas/amostra obtido na profundidade de $6 \mathrm{~cm}$ foi significativamente maior do que na de $3 \mathrm{~cm}$ e intermediário na de $9 \mathrm{~cm}$ (Tabela 1).

A comparação das cultivares quanto à média do número de larvas obtido nas três profundidades de amostragem apontou a 'BRS Querência' e 'IRGA 417' como as mais infestadas, diferindo significativamente das restantes; a comparação das profundidades de amostragem quanto à media do número de larvas obtido nas seis cultivares indicou um índice de infestação significativamente maior em amostras retiradas aos $6 \mathrm{~cm}$ do que aos $3 \mathrm{~cm}$, mantendo-se as amostras de $9 \mathrm{~cm}$ com uma infestação intermediária (Tabela 1).

As cultivares diferiram significativamente quanto ao número de adultos de $\boldsymbol{O}$. oryza $\boldsymbol{e}$ obtido pelas amostragens às profundidades de $6 \mathrm{e} 9 \mathrm{~cm}$, sendo a 'BRS Firmeza', em ambos os casos, a menos infestada. Também ocorreu interação entre profundidade de amostragem e cultivar em relação ao número de adultos, porém, apenas na 'BRS Querência', sendo o número de adultos/amostra obtido na profundidade de $6 \mathrm{~cm}$ significativamente maior do que na de $3 \mathrm{~cm}$ e intermediário na de $9 \mathrm{~cm}$ (Tabela 1).

A comparação das cultivares quanto à média do número de adultos oriundos de amostras retiradas nas três profundidades apontou a 'BRS Querência' e a 'BRS Sinuelo CL' como as mais infestadas, diferindo significativamente da 'BRS

Tabela 1 - Número de larvas e adultos de Oryzophagus oryzae em amostras de solo e raízes coletadas às profundidades de 3,6 e $9 \mathrm{~cm}$, em parcelas experimentais de seis cultivares de arroz. Capão do Leão - RS. 2009/10.

\begin{tabular}{|c|c|c|c|c|}
\hline \multirow{2}{*}{ Cultivares } & \multicolumn{4}{|c|}{--1ensetos/profundidade de amostragem ${ }^{1,2}$} \\
\hline & $3 \mathrm{~cm}$ & $6 \mathrm{~cm}$ & $9 \mathrm{~cm}$ & Média \pm EP \\
\hline & . & -----Larvas--- & 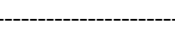 & 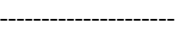 \\
\hline 'BRS Atalanta' & 16,8 a A & 26,3 a $A$ & 19,5 a A & $20,8 \pm 2,0 \mathrm{~b}$ \\
\hline 'BRS Firmeza' & 15,5 a A & 23,5 a A & 18,2 a $\mathrm{A}$ & $19,1 \pm 1,9 \mathrm{~b}$ \\
\hline 'BRS Ligeirinho’ & 16,8 a A & 20,3 a A & 20,2 a $\mathrm{A}$ & $19,1 \pm 1,7 \mathrm{~b}$ \\
\hline 'BRS Querência' & 23,7 a A & 39,2 a $\mathrm{A}$ & 35,8 a $\mathrm{A}$ & $32,9 \pm 3,1 \mathrm{a}$ \\
\hline 'BRS Sinuelo CL' & 13,8 a B & 27,2 a A & $20,5 \mathrm{a} \mathrm{AB}$ & $20,5 \pm 2,2 b$ \\
\hline 'IRGA 417' & 27,3 a $\mathrm{A}$ & 36,8 a A & 36,8 a $\mathrm{A}$ & $33,7 \pm 4,7 \mathrm{a}$ \\
\hline Média \pm EP & $19,0 \pm 1,6 \mathrm{~B}$ & $28,9 \pm 2,3 \mathrm{~A}$ & $25,1 \pm 2,2 \mathrm{AB}$ & $24,3 \pm 2,2$ \\
\hline 'BRS Atalanta' & 12,0 a A & $22,5 \mathrm{ab} \mathrm{A}$ & $16,3 \mathrm{ab} A$ & $16,9 \pm 1,9 \mathrm{bc}$ \\
\hline 'BRS Firmeza' & 13,0 a A & $18,8 \mathrm{~b} \mathrm{~A}$ & 14,3 b A & $15,5 \pm 2,3 \mathrm{c}$ \\
\hline 'BRS Ligeirinho’' & 30,2 a A & $32,0 \mathrm{ab} \mathrm{A}$ & $25,0 \mathrm{ab} A$ & $29,0 \pm 3,1 \mathrm{ab}$ \\
\hline 'BRS Querência' & 24,0 a B & 43,3 a A & 38,2 a $A B$ & $35,2 \pm 4,6 \mathrm{a}$ \\
\hline 'BRS Sinuelo CL' & 32,3 a A & $32,5 \mathrm{ab} A$ & $25,5 \mathrm{ab} A$ & $30,1 \pm 3,6 \mathrm{a}$ \\
\hline 'IRGA 417' & 15,3 a A & $27,0 \mathrm{ab} \mathrm{A}$ & $28,7 \mathrm{ab} A$ & $23,7 \pm 3,3 \mathrm{abc}$ \\
\hline Média \pm EP & $21,2 \pm 2,3 \mathrm{~B}$ & $29,4 \pm 2,9 \mathrm{~A}$ & $24,7 \pm 2,2 \mathrm{AB}$ & $25,5 \pm 2,5$ \\
\hline
\end{tabular}

${ }^{1}$ Total de insetos em quatro amostras/parcela, coletadas aos 33 e 39 dias pós-irrigação por inundação das cultivares, para a contagem de larvas e adultos, respectivamente.

${ }^{2}$ Valores seguidos da mesma letra, minúscula na coluna e maiúscula na linha, não diferem significativamente pelo teste de Tukey em nível de $5 \%$ de probabilidade; o CV para o número de larvas e de adultos foi, respectivamente, de $22,2 \%$ e $23,6 \%$.

Ciência Rural, v.41, n.12, dez, 2011. 
Atalanta' e 'BRS Firmeza'. A comparação entre as profundidades de amostragem com base na média do número de adultos obtido nas seis cultivares também indicou uma infestação significativamente maior em amostras retiradas aos $6 \mathrm{~cm}$ do que aos $3 \mathrm{~cm}$, mantendose uma quantidade intermediária de insetos nas amostras de $9 \mathrm{~cm}$ (Tabela 1).

O menor número de larvas e de adultos de O. oryzae em amostras de $3 \mathrm{~cm}$ do Planossolo Háplico (Tabela 1) pode ter decorrido da não captura de larvas situadas mais profundamente. No entanto, a deteç̧ão de maior número de larvas e de adultos nas amostras de $6 \mathrm{~cm}$ ajusta-se à indicação de profundidade de amostragem para a avaliação da população larval de $\boldsymbol{L}$. oryzophilus em solos argilosos, que é de 5 a $7,5 \mathrm{~cm}$ (JOHNSON et al., 2006).

O índice intermediário de infestação larval de $\boldsymbol{O}$. oryzae detectado em amostras com $9 \mathrm{~cm}$, mesmo não tendo diferido significativamente do observado nas amostras de $6 \mathrm{~cm}$ (Tabela 1), pode ter resultado de uma maior dificuldade para visualizar larvas menores (1ํ e 2o instar). Quando as amostras são sacudidas submersas, forma-se uma mistura de água, solo e pedaços de raízes no interior das peneiras utilizadas para contagem das larvas, conforme ressaltado por MARTINS et al. (2001). Assim sendo, evidencia-se uma maior dificuldade para a visualização de larvas, tratandose de amostras retiradas a $9 \mathrm{~cm}$ de profundidade, devido estas carrearem maior volume de solo, raízes e detritos às peneiras.

As diferenças significativas quanto ao número de adultos de $\boldsymbol{O}$. oryzae que emergiram das amostras intactas de $3,6 \mathrm{e} 9 \mathrm{~cm}$ (Tabela 1) podem também estar associadas à profundidade de amostragem. Por outro lado, podem estar associadas aos efeitos que as plantas de arroz exercem sobre a biologia do inseto, principalmente, considerando que há diferentes tipos de resistência, incluindo antibiose (MARTINS \& TERRES, 1995). Nesse sentido, foi observado que, enquanto nas cultivares 'BRS Ligeirinho' e 'BRS Sinuelo CL' o número de adultos emergidos/amostra intacta foi significativamente superior ao de larvas/ amostra desintegrada, na cultivar 'IRGA 417' o número de adultos foi significativamente inferior ao de larvas/ amostra (Tabela 2). Nas cultivares 'BRS Atalanta', 'BRS Firmeza' e 'BRS Querência' não ocorreu diferença significativa entre o número de adultos e de larvas/ amostra. Esse método de comparar o número de larvas e de adultos em amostras de solo e raízes, desintegradas e intactas, respectivamente, foi usado para avaliar a resistência de genótipos de arroz a $\boldsymbol{O}$. oryzae, evidenciando efeitos de antibiose durante a fase larval (MARTINS et al., 2001).

Considerando que atualmente o elevado custo e a escassez de mão de obra disponível para ser empregada nos arrozais podem dificultar a adoção de boas práticas de manejo, como o monitoramento da população larval de $\boldsymbol{O}$. oryzae, a utilização de técnicas de amostragem do inseto que requerem a retirada de amostras em menores profundidades do solo pode tornar o processo mais rápido e preciso, portanto, aumentando sua eficácia.

Tabela 2 - Comparação entre o número de larvas e de adultos de Oryzophagus oryzae em amostras de solo e raízes coletadas às profundidades de 3, 6 e 9cm, em parcelas experimentais de seis cultivares de arroz. Capão do Leão - RS. 2009/10.

\begin{tabular}{|c|c|c|c|c|c|c|c|c|c|}
\hline \multirow[t]{2}{*}{ Cultivares } & \multicolumn{2}{|c|}{--------3cm-------- } & \multicolumn{2}{|c|}{---------6cm--------- } & \multicolumn{2}{|c|}{---------9cm--------- } & \multicolumn{2}{|c|}{---------Média--------- } & \multirow{2}{*}{$\mathrm{V}(\%)^{3}$} \\
\hline & Larva & Adulto & Larva & Adulto & Larva & Adulto & Larva & Adulto & \\
\hline 'BRS Atalanta' & $16,8 \mathrm{~A}$ & $12,0 \mathrm{~A}$ & $26,3 \mathrm{~A}$ & $22,5 \mathrm{~A}$ & $19,5 \mathrm{~A}$ & $16,3 \mathrm{~A}$ & $20,8 \mathrm{~A}$ & $16,9 \mathrm{~A}$ & $-18,8$ \\
\hline 'BRS Firmeza' & $15,5 \mathrm{~A}$ & $13,0 \mathrm{~A}$ & $23,5 \mathrm{~A}$ & $18,8 \mathrm{~A}$ & $18,2 \mathrm{~A}$ & $14,3 \mathrm{~A}$ & $19,1 \mathrm{~A}$ & $15,5 \mathrm{~A}$ & $-18,8$ \\
\hline 'BRS Ligeirinho' & $16,8 \mathrm{~B}$ & $30,2 \mathrm{~A}$ & $20,3 \mathrm{~A}$ & $32,0 \mathrm{~A}$ & $20,2 \mathrm{~A}$ & $25,0 \mathrm{~A}$ & $19,1 \mathrm{~B}$ & $29,0 \mathrm{~A}$ & $+51,8$ \\
\hline 'BRS Querência' & $23,7 \mathrm{~A}$ & $24,0 \mathrm{~A}$ & $39,2 \mathrm{~A}$ & $43,3 \mathrm{~A}$ & $35,8 \mathrm{~A}$ & $38,2 \mathrm{~A}$ & $32,9 \mathrm{~A}$ & $35,2 \mathrm{~A}$ & $+7,0$ \\
\hline 'BRS Sinuelo CL' & $13,8 \mathrm{~B}$ & $32,3 \mathrm{~A}$ & $27,2 \mathrm{~A}$ & $32,5 \mathrm{~A}$ & $20,5 \mathrm{~A}$ & $25,5 \mathrm{~A}$ & $20,5 \mathrm{~B}$ & $30,1 \mathrm{~A}$ & $+46,8$ \\
\hline 'IRGA 417' & $27,3 \mathrm{~A}$ & $15,3 \mathrm{~A}$ & $36,8 \mathrm{~A}$ & $27,0 \mathrm{~A}$ & $36,8 \mathrm{~A}$ & $28,7 \mathrm{~A}$ & $33,7 \mathrm{~A}$ & $23,7 \mathrm{~B}$ & $-29,7$ \\
\hline Média & $19,0 \mathrm{~A}$ & $21,2 \mathrm{~A}$ & $28,9 \mathrm{~A}$ & $29,4 \mathrm{~A}$ & $25,1 \mathrm{~A}$ & $24,7 \mathrm{~A}$ & $24,3 \mathrm{~A}$ & $25,5 \mathrm{~A}$ & $+4,9$ \\
\hline
\end{tabular}

${ }^{1}$ Total de insetos em quatro amostras/parcela, coletadas aos 33 e 39 dias pós-irrigação por inundação das cultivares, para a contagem de larvas e adultos, respectivamente.

${ }^{2}$ Valores seguidos da mesma letra, minúscula na coluna e maiúscula na linha, não diferem significativamente pelo teste de Tukey em nível de $5 \%$ de probabilidade $(\mathrm{CV}=25,8 \%)$.

${ }^{3}$ Variação entre a média do número de larvas (L) e de adultos (A), obtidos aos 33 dias e até 69 dias pós-inundação das parcelas, respectivamente; $\mathrm{V}=[(\mathrm{L}-\mathrm{A}) / \mathrm{L}) \times 100]$. 


\section{CONCLUSÃO}

Amostras cilíndricas de solo e raízes de arroz retiradas em Planossolo Háplico à profundidade de $6 \mathrm{~cm}$ são mais apropriadas à captura e à visualização de larvas de $\boldsymbol{O}$. oryzae , na suspensão (solo e água) formada nas peneiras usadas para a sua contagem, como também à obtenção de adultos desse inseto.

Variações no número de adultos de O. oryzae que emergem de amostras cilíndricas de solo e raízes de arroz não dependem exclusivamente do número de larvas nessas amostras, mas também do grau de resistência ao inseto da cultivar envolvida.

\section{REFERÊNCIAS}

CARBONARI, J.J. et al. Relação entre flutuação populacional de Oryzophagus oryzae (Costa Lima) (Coleoptera: Curculionidae) e período de perfilhamento de cultivares de arroz Irrigado. Anais da Sociedade Entomológica do Brasil, v.2, p.361-366, 2000. Disponível em: <http://www.scielo.br/ scielo.php?script=sci_arttext \& pid=S0301$80592000000200019 \& \operatorname{lng}>$. Acesso em: 10 jun. 2010. doi: 10.1590/S0301-80592000000200019.

CONGRESSO BRASILEIRO DE ARROZ IRRIGADO, 5., 2007, Pelotas, RS. Arroz irrigado: recomendações técnicas da pesquisa para o sul do Brasil. Pelotas: SOSBAI, Embrapa Clima Temperado, 2007. 164p.

GRÜTZMACHER, A.D. et al. Viabilidade da antecipação do tratamento de sementes de arroz com inseticidas em relação à data de semeadura no controle de Oryzophagus oryzae (Coleoptera: Curculionidae). Ciência Rural, v.38, p.18301835, 2008. Disponível em: <http://www.scielo.br/ scielo.php ? script $=$ sci arttext \& pid = S 0103 $84782008000700005 \& \operatorname{lng}>$. Acesso em: 5 jul. 2010. doi: 10.1590/S0103-84782008000700005.

HOFFMANN-CAMPO, C.B. et al. Ciclo biológico, comportamento e distribuição estacional de Sternechus subsignatus Boheman, 1836 (Col.: Curculionidae) em soja, no norte do Paraná. In: EMBRAPA. Resultados de pesquisa de soja. Londrina, PR: EMBRAPA/ CNPSo, 1988/89. 1989. p.64-69.

JOHNSON, D.R. et al. Management of the insect pests of rice. Arkansas: University of Arkansas Division of Agriculture, 2006. Online. Disponível em: <http://www.aragriculture.org/ insects/rice/rice_water_weevil.htm>. Acesso em: 10 ago. 2010.

MARTINS, J.F. da S.; CUNHA, U.S. da. Situação do sistema de controle químico do gorgulho-aquático Oryzophagus oryzae (Costa Lima) (Coleoptera: Curculionidae) na cultura do arroz no Rio Grande do Sul. Pelotas: Embrapa Clima Temperado, 2007. 25p. (Embrapa Clima Temperado. Documentos, 215).

MARTINS, J.F. da S.; PRANDO, H.F. Bicheira-da-raiz-do-arroz. In. SALVADORI, J.R. (Ed.). Pragas de solo no Brasil. Passo Fundo: Embrapa Trigo; Dourados: Embrapa Agropecuária Oeste; Cruz Alta: Fundacep-Fecotrigo, 2004. p.259-296.

MARTINS, J.F. da S.; TERRES, A.L.S. Avaliação de germoplasma de arroz visando resistência à Oryzophagus oryzae (Costa Lima). Anais da Sociedade Entomológica do Brasil, v.24, p.445-453, 1995.

MARTINS, J.F. da S. et al. Descrição e manejo integrado de insetos-praga em arroz irrigado. In: GOMES, A. da S.; MAGAlHÃES Jr., A.M. Arroz irrigado no Sul do Brasil. Brasília, DF: Embrapa Informação Tecnológica, 2004. Cap.19, p.635-675

MARTINS, J.F. da S. et al. Novo método para aferição da densidade populacional do gorgulho-aquático em plantas de arroz irrigado. Agropecuária Clima Temperado, v.4, p.363-370, 2001.

MOREIRA, G.R.P. Oviposition by the rice infesting weevil, Oryzophagus oryzae (Costa Lima, 1936) (Coleoptera, Curculionidae): influence of water depth and host-plant characteristics. Revista Brasileira de Zoociências, v.4, p.237-253, 2002.

OLIVEIRA, L.J. et al. Seasonal and vertical distribution of Phyllophaga cuyabana (Moser) (Coleoptera: Melolonthidae) in the Soil Profile. Neotropical Entomology, v.5, p.582588, 2009. Disponível em: <http://www.scielo.br/ scielo.php? script =sci_abstract\&pid=S 1519 $566 X 2009000500004 \& \operatorname{lng}=$ en $\& n r m=i s o \& t \operatorname{lng}=p t>$. Acesso em: 20 jul. 2010. doi: 10.1590/S1519-566X2009000500004.

SILVA, M.T.B. Aspectos ecológicos de Sternechus subsignatus Boheman (Coleoptera: Curculionidae) em soja no plantio direto. Anais da Sociedade Entomológica Brasil, v.27, p.47-53, 1998.

SILVA, F. de A. S. Assistat 7.5 Beta. Campina Grande: Departamento de Engenharia Agrícola do Centro de Tecnologia e Recursos Naturais da Universidade Federal de Campina Grande, 2008. Online. Disponível em: <http://www.assistat.com/ indexp.html>. Acesso em: 20 mar. 2010.

SILVA, F.F. et al. Avaliação da resistência de arroz a Oryzophagus oryzae com e sem chance de escolha da planta hospedeira. Revista Brasileira de Agrociência, v.9, p.135-140, 2003.

TUGWELL, W.P.; STEPHEN, F.M. Rice water weevil seasonal abundance, economic levels and sequential sampling plant. Fayeltville: Agricultural Experiment Station, 1981. 16p. (Bulletin n.849). 